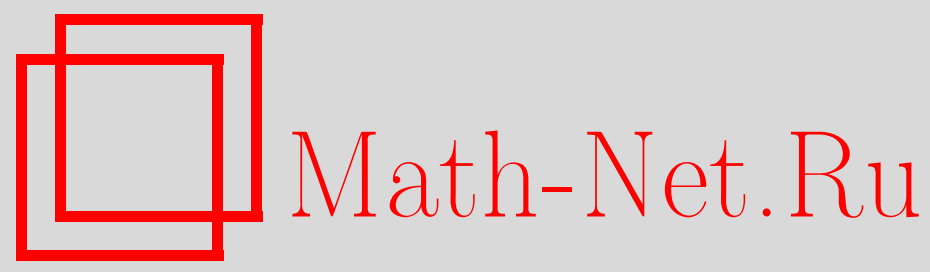

В. С. Пилиди, Обобщенная фредгольмовость и априорные оценки для линейных операторов в тензорных произведениях гильбертовых пространств, Матем. заметки, 1998, том 64, выпуск 6, 902-912

DOI: https://doi.org/10.4213/mzm1469

Использование Общероссийского математического портала Math-Net.Ru подразумевает, что вы прочитали и согласны с пользовательским соглашением http://www.mathnet.ru/rus/agreement

Параметры загрузки:

IP: 18.207 .199 .55

26 апреля 2023 г., 12:38:46

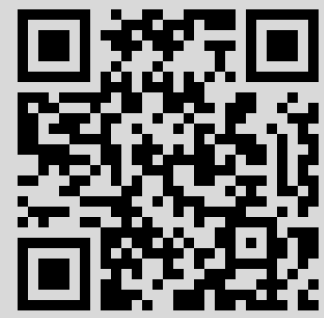




\title{
ОБОБЩЕННАЯ ФРЕДГОЛЬМОВОСТЬ И АПРИОРНЫЕ ОЦЕНКИ ДЛЯ ЛИНЕЙНЫХ ОПЕРАТОРОВ В ТЕНЗОРНЫХ ПРОИЗВЕДЕНИЯХ ГИЛЬБЕРТОВЫХ ПРОСТРАНСТВ
}

\section{В. С. Пилиди}

\begin{abstract}
Для линейного непрерывного оператора, действующего в гильбертовом пространстве, доказывается равносильность обобщенной фредгольмовости (обратимости по модулю некоторого идеала) и наличия некоторых априорных оценок. Этот результат применяется к доказательству утверждений о связи $n$-, $d$-нормальности и фредгольмовости (обобщенной и обычной) линейных операторов, действующих в тензорных произведениях гильбертовых пространств, с наличием соответствующих априорных оценок.

Библиография: 6 названий.
\end{abstract}

Напомним, что линейный непрерывный оператор $A$, действуюший в банаховом пространстве $\mathfrak{X}$, назьвается $n$-нормальныц $(d$-нормальныл), если его образ замкнут и подпространство $\operatorname{ker} A$ (соответственно $\operatorname{ker} A^{*}$ ) конечномерно. Оператор называется $\Phi$-оператором (фредгольмовыц м оператором), если он $n$ - и $d$-нормален (в литературе используется и несколько иное определение фредгольмова оператора $[1, \S 14])$.

Пусть $\mathscr{B}(\mathfrak{X})(\mathscr{K}(\mathfrak{X}))$ - множество всех линейных непрерывных операторов, действуюших в пространстве $\mathfrak{X}$. Фредгольмовость оператора $A$ равносильна обратимости смежного класса $A+\mathscr{K}(\mathfrak{X}) \in \mathscr{B}(\mathfrak{X}) / \mathscr{K}(\mathfrak{X})$. Если $\mathfrak{X}$ - гильбертово пространство, то $n$-нормальность ( $d$-нормальность) оператора $A$ равносильна обратимости этого смежного класса слева (справа).

Указанное свойство позволяет обобщить понятие фредгольмовости, связывая ее с идеалом, отличньм от множества $\mathscr{K}(\mathfrak{X})$. Из работ в этом направлении упомянем статью [2], где исследуются идеалы, связанные с обобщенным условием Реллиха (приводимое ниже условие Б) ), и ассоциированная с ними обобщенная фредгольмовость. В работе [3] обобщенная фредгольмовость использована для исследования обычной фредгольмовости бисингулярных операторов.

Предположим, что банахово пространство $\mathfrak{X}$ компактно вложено в банахово пространство $\mathfrak{Y}$. Известно (см., например, $[1, \S 7])$, что $n$-нормальность оператора $A$ равносильна тому, что вьполняется априорная оценка

$$
\|x\|_{\mathfrak{X}} \leqslant \operatorname{const}\left(\|A x\|_{\mathfrak{X}}+\|x\|_{\mathfrak{Y}}\right), \quad x \in \mathfrak{X} .
$$

Аналогичная оценка для оператора $A^{*}$ равносильна $d$-нормальности оператора $A$.

Работа выполнена при поддержке Российского фонда фундаментальных исследований, грант № 96-01-01195. 
В настоящей работе мы распространяем этот результат на случай обобщенной фредгольмовости линейных операторов, действующих в гильбертовом пространстве. В п. 1 вводится понятие обобщенной фредгольмовости и устанавливается связь между этим понятием и наличием априорньх оценок. Полученный здесь результат используется в п. 2 для доказательства равносильности свойств $n$-, $d$-нормальности, фредгольмовости (обобщенной и обычной) и наличия смешанных априорных оценок для операторов некоторого класса. Отметим, что применяемая в доказательстве леммы 2 конструкция изоморфного вложения опирается на схему, предложенную в статье [4] для построения изометрического представления факторалгебры $\mathscr{B}(\mathfrak{X}) / \mathscr{K}(\mathfrak{X})($ алгебры Калкина) в случае гильбертова пространства $\mathfrak{X}$.

1. Всюду далее буквой $H$ (с индексами или без них) обозначаются сепарабельные бесконечномерные комплексные гильбертовы пространства. Напомним, что любые два таких пространства $H_{1}$ и $H_{2}$ изометрически изоморфны, т.е. существует изометрический обратимьй линейный оператор $U: H_{1} \rightarrow H_{2}$.

Всюду в этом пункте предполагается, что $\mathfrak{A}(\subset \mathscr{B}(H))$ - некоторая $C^{*}$-алгебра, содержащая единичньй оператор, $\mathfrak{K}(\subset \mathfrak{A})$ - собственный замкнутый двусторонний *-идеал.

ОПРЕДЕЛЕНИЕ. Оператор $A \in \mathfrak{A}$ назовем $\Phi_{\mathfrak{K}}$-оператором, если смежный класс $A+\mathfrak{K} \in \mathfrak{A} / \mathfrak{K}$ обратим.

Если $\mathfrak{A}_{1} \supset \mathfrak{A}-C^{*}$-алгебра, в которой $\mathfrak{K}$ является двусторонним идеалом, и $A \in \mathfrak{A}$, то смежный класс $A+\mathfrak{K}$ обратим в $\mathfrak{A}_{1} / \mathfrak{K}$ тогда и только тогда, когда он обратим в $\mathfrak{A} / \mathfrak{K}$ [5, теорема 2.4.11]. Иначе говоря, $\Phi_{\mathfrak{K}}$-свойство оператора $A$ не зависит от содержащей этот оператор $C^{*}$-алгебры, в которой $\mathfrak{K}$ является двусторонним идеалом.

Нам потребуется аналогичное утверждение для односторонней обратимости смежного класса $A+\mathfrak{K}$.

Лемма 1. Пусть $\mathfrak{A}_{1}-C^{*}$-алгебра с единичей, $\mathfrak{A}_{0}-$ ее $C^{*}{ }_{-}$подалгебра с той жсе единиией. Если әлемент $а \in \mathfrak{A}_{0}$ односторонне обратим в $\mathfrak{A}_{1}$, то он обратим $c$ той же стороны в $\mathfrak{A}_{0}$.

ДокАЗАТЕЛЬСтво. Очевидно, можно ограничиться случаем обратимости слева и предполагать, что $\mathfrak{A}_{1}=\mathscr{B}(\mathscr{H})$, где $\mathscr{H}$ - некоторое гильбертово пространство. Предположим, что оператор $A$ обратим слева в $\mathfrak{A}_{1}$. Это равносильно тому, что вьполняется оценка

$$
\|A x\| \geqslant c\|x\|, \quad x \in \mathscr{H},
$$

с некоторой константой $c>0$. Рассмотрим полярное разложение оператора $A$ (см. [5, теорема 2.3.4]): $A=U P$, где $P=\left(A^{*} A\right)^{1 / 2}(\geqslant 0), U$ - частичная изометрия, $\operatorname{ker} U=\operatorname{ker} A$. Из (1) следует, что $\operatorname{ker} A=\{0\}$. Поэтому $U-$ изометрия и $U^{*} U=I$. Для любого $x \in \mathscr{H}$

$$
\|P x\|^{2}=\left(\left(A^{*} A\right)^{1 / 2} x,\left(A^{*} A\right)^{1 / 2} x\right)=\left(A^{*} A x, x\right)=\|A x\|^{2} .
$$

Поэтому для оператора $P$ выполняется оценка вида (1); следовательно, он обратим. Учтем, что $P \in \mathfrak{A}_{0}$. Тогда $P^{-1} \in \mathfrak{A}_{0}$ (см. [5, теорема 2.4.11]). Теперь из равенства $U=A P^{-1}$ вытекает, что $U \in \mathfrak{A}_{0}$. Тогда для оператора $B=P^{-1} U^{*}\left(\in \mathfrak{A}_{0}\right)$ имеем $B A=I$. Лемма доказана.

Пока будем предполагать, что выполнено такое условие:

A) $\bigcap_{T \in \mathfrak{K}} \operatorname{ker} T=\{0\}$. 
Ниже на идеал $\mathfrak{K}$ будут наложены дополнительные ограничения.

Пусть $x, x_{n}(n=1,2, \ldots)$ - элементы из $H$. Запись

$$
\underset{n \rightarrow \infty}{\mathfrak{K}-\lim } x_{n}=x
$$

означает, что для любого $T \in \mathfrak{K}$

$$
\lim _{n \rightarrow \infty}\left\|T\left(x_{n}-x\right)\right\|=0
$$

В силу условия А) последнему равенству удовлетворяет не более одного элемента $x$.

Напомним теперь определение обобщенного предела (подробности, включая существование обобщенного предела, см., например, в [6, гл. 3]). Как обычно, через $\ell^{\infty}$ обозначаем банахово пространство ограниченных последовательностей комплексных чисел c sup-нормой. Через $\ell_{\mathbb{R}}^{\infty}$ обозначим аналогичное пространство последовательностей вешественньх чисел. Обобщенный предел - это линейный непрерьвньй функционал $\operatorname{Lim}_{n \rightarrow \infty} x_{n}$, определенньй на пространстве $\ell_{\mathbb{R}}^{\infty}$ и обладающий свойствами:

1) если $\left\{x_{n}\right\},\left\{y_{n}\right\} \in \ell_{\mathbb{R}}^{\infty}$ и $x_{n} \leqslant y_{n}$ для всех $n$, то

$$
\operatorname{Lim}_{n \rightarrow \infty} x_{n} \leqslant \operatorname{Lim}_{n \rightarrow \infty} y_{n}
$$

2) для всех $\left\{x_{n}\right\} \in \ell_{\mathbb{R}}^{\infty}$

$$
\varliminf_{n \rightarrow \infty} x_{n} \leqslant \operatorname{Lim}_{n \rightarrow \infty} x_{n} \leqslant \varlimsup_{n \rightarrow \infty} x_{n}
$$

Из свойства 2) следует, что если $\left\{x_{n}\right\} \in \ell_{\mathbb{R}}^{\infty}$ и существует $\lim _{n \rightarrow \infty} x_{n}$, то

$$
\operatorname{Lim}_{n \rightarrow \infty} x_{n}=\lim _{n \rightarrow \infty} x_{n}
$$

$\Phi$ ункционал Lim очевидным образом распространяется на пространство $\ell^{\infty}$.

Обозначим через $\ell_{0}^{\infty}(H, \mathfrak{K})$ множество всех ограниченных по норме последовательностей $\left\{x_{n}\right\}_{n=1}^{\infty}$ элементов пространства $H$ таких, что

$$
\underset{n \rightarrow \infty}{\mathfrak{K}-\lim } x_{n}=0
$$

Это множество становится линейным пространством, если операции ввести равенствами:

$$
\left\{x_{n}\right\}+\left\{y_{n}\right\}=\left\{x_{n}+y_{n}\right\}, \quad \alpha\left\{x_{n}\right\}=\left\{\alpha x_{n}\right\}, \quad\left\{x_{n}\right\},\left\{y_{n}\right\} \in \ell_{0}^{\infty}(H, \mathfrak{K}), \quad \alpha \in \mathbb{C} .
$$

Потребуем дополнительно, чтобы вьполнялось следующее условие:

Б) существует последовательность $\left\{R_{n}\right\}_{n=1}^{\infty}$ ортопроекторов из идеала $\mathfrak{K}$ такая, что для любого $A \in \mathfrak{K}$

$$
\lim _{n \rightarrow \infty}\left\|A-A R_{n}\right\|=0
$$


ЗАмЕчАниЕ. В приводимых в п. 2 приложениях полученных здесь результатов операторы $R_{n}$ при $n \rightarrow \infty$ сильно сходятся к единичному оператору. Отсюда немедленно вытекает, что идеал $\mathfrak{K}$ обладает свойством А).

Справедливо утверждение.

Лемма 2. Если $A \in \mathfrak{A} и$ для любой последовательности $\left\{x_{n}\right\} \in \ell_{0}^{\infty}(H, \mathfrak{K})$ выполняется равенство

$$
\lim _{n \rightarrow \infty}\left\|A x_{n}\right\|=0
$$

$m o A \in \mathfrak{K}$.

ДокАЗАТЕЛЬСТво. Покажем, что выполнено (2). Отсюда, учитьвая, что для всех $n$ $A R_{n} \in \mathfrak{K}$, получим требуемое утверждение. Допустим противное, т.е.

$$
\delta \stackrel{\text { def }}{=} \varlimsup_{n \rightarrow \infty}\left\|A-A R_{n}\right\|>0
$$

Возьмем последовательность $\left\{y_{n}\right\}_{n=1}^{\infty}$ элементов из $H$ такую, что $\left\|y_{n}\right\|=1$ для всех $n$ и

$$
\varlimsup_{n \rightarrow \infty}\left\|\left(A-A R_{n}\right) y_{n}\right\|=\delta .
$$

Положим $x_{n}=\left(I-R_{n}\right) y_{n}, n=1,2, \ldots$ Из условия Б) следует, что $\left\{x_{n}\right\} \in \ell_{0}^{\infty}(H, \mathfrak{K})$. Переписьвая предыдущее равенство в виде

$$
\varlimsup_{n \rightarrow \infty}\left\|A x_{n}\right\|=\delta(>0)
$$

получаем противоречие с условием. Лемма доказана.

Определим в пространстве $\ell_{0}^{\infty}(H, \mathfrak{K})$ полуторалинейную форму $\langle\cdot, \cdot\rangle$ формулой

$$
\left\langle\left\{x_{n}\right\},\left\{y_{n}\right\}\right\rangle=\operatorname{Lim}_{n \rightarrow \infty}\left(x_{n}, y_{n}\right) \text {. }
$$

Правая часть этой формулы определена корректно ввиду ограниченности последовательности $\left\{\left(x_{n}, y_{n}\right)\right\}_{n=1}^{\infty}$. Форма $\langle\cdot, \cdot\rangle$ обладает всеми свойствами скалярного произведения, кроме положительной определенности, являясь положительно полуопределенной. Построим из линейного пространства $\ell_{0}^{\infty}(H, \mathfrak{K})$ гильбертово пространство следующим стандартньм способом.

Множество $\mathfrak{N}$ всех элементов $\left\{x_{n}\right\} \in \ell_{0}^{\infty}(H, \mathfrak{K})$, удовлетворяющих условию

$$
\operatorname{Lim}_{n \rightarrow \infty}\left\|x_{n}\right\|=0
$$

является подпространством линейного пространства $\ell_{0}^{\infty}(H, \mathfrak{K})$. Введем скалярное произведение в факторпространстве $\ell_{0}^{\infty}(H, \mathfrak{K}) / \mathfrak{N}$ :

$$
\left(\left\{x_{n}\right\}+\mathfrak{N},\left\{y_{n}\right\}+\mathfrak{N}\right)=\left\langle\left\{x_{n}\right\},\left\{y_{n}\right\}\right\rangle \text {. }
$$

Очевидно, что правая часть этой формулы не зависит от выбора представителей смеж-

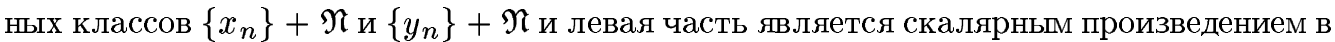
линейном пространстве $\ell_{0}^{\infty}(H, \mathfrak{K}) / \mathfrak{N}$. Обозначим через $\mathbb{H}$ пополнение этого предгильбертова пространства. 
Пусть $A \in \mathfrak{A}$. Если $\left\{x_{n}\right\} \in \ell_{0}^{\infty}(H, \mathfrak{K})$, то $\left\{A x_{n}\right\} \in \ell_{0}^{\infty}(H, \mathfrak{K})$. Из оценки

$$
\operatorname{Lim}_{n \rightarrow \infty}\left\|A x_{n}\right\| \leqslant\|A\| \cdot \operatorname{Lim}_{n \rightarrow \infty}\left\|x_{n}\right\|
$$

вытекает, что линейньй оператор $\widehat{A}: \ell_{0}^{\infty}(H, \mathfrak{K}) / \mathfrak{N} \rightarrow \ell_{0}^{\infty}(H, \mathfrak{K}) / \mathfrak{N}$, действующий по правилу

$$
\widehat{A}:\left\{x_{n}\right\}+\mathfrak{N} \mapsto\left\{A x_{n}\right\}+\mathfrak{N},
$$

определен корректно и непрерьвен. Продолжение по непрерьвности этого оператора на все пространство $\mathbb{H}$ также будем обозначать через $\widehat{A}$. Отметим, что $\|\widehat{A}\| \leqslant\|A\|$. Мы доказали, что отображение $\Xi: A \mapsto \widehat{A}$, действующее из $\mathfrak{A}$ в $\mathscr{B}(\mathbb{H})$, является непрерьвным. Справедливо более точное утверждение.

Лемма 3. Отображсние $\Xi: \mathfrak{A} \rightarrow \mathscr{B}(\mathbb{H})$ является непрерывным *-гомоморфизмом, $\operatorname{ker} \Xi=\mathfrak{K}$, и индуцированный гомоморфизм $\widehat{\Xi}: \mathfrak{A} / \mathfrak{K} \rightarrow \mathscr{B}(\mathbb{H})$ является изометрически *-изоморфным вложсением.

ДокАЗАтЕльство. Очевидно, что отображение $\Xi$ является *-гомоморфизмом. Его непрерьвность была отмечена вьше.

Рассмотрим ker $\Xi$. Если $A \in \mathfrak{K}$, то для любой последовательности $\left\{x_{n}\right\} \in \ell_{0}^{\infty}(H, \mathfrak{K})$ выполнено (3), т.е. $\left\{A x_{n}\right\} \in \mathfrak{N}$. Поэтому $\widehat{A}=0$ и $\mathfrak{K} \subset \operatorname{ker} \Xi$.

Пусть $A \in \operatorname{ker} \Xi$, т.е. $A \in \mathfrak{A}$ и $\widehat{A}=0$. Последнее означает, что для любой последовательности $\left\{x_{n}\right\} \in \ell_{0}^{\infty}(H, \mathfrak{K})$

$$
\operatorname{Lim}_{n \rightarrow \infty}\left\|A x_{n}\right\|=0
$$

Покажем, что при этом вьполнено (3). Действительно, допустим, что это не так. Тогда

$$
\delta \stackrel{\text { def }}{=} \varlimsup_{n \rightarrow \infty}\left\|A x_{n}\right\|>0
$$

Выберем подпоследовательность $\left\{x_{n_{k}}\right\}_{k=1}^{\infty}$ такую, что

$$
\lim _{k \rightarrow \infty}\left\|A x_{n_{k}}\right\|=\delta
$$

Мы получили противоречие, поскольку $\left\{x_{n_{k}}\right\}_{k=1}^{\infty} \in \ell_{0}^{\infty}(H, \mathfrak{K})$,

$$
\operatorname{Lim}_{k \rightarrow \infty}\left\|A x_{n_{k}}\right\|=\lim _{k \rightarrow \infty}\left\|A x_{n_{k}}\right\|=\delta \neq 0
$$

Из леммы 2 следует, что $A \in \mathfrak{K}$, т.e. $\operatorname{ker} \Xi \subset \mathfrak{K}$ и, окончательно, $\operatorname{ker} \Xi=\mathfrak{K}$.

Утверждение об изометричности индуцированного *-изоморфного вложения является известньм результатом теории алгебр [5, теорема 3.1.5]. Лемма доказана.

Пусть $L_{1}$ и $L_{2}$ - линейные пространства над одним полем. Напомним, что вложениeм пространства $L_{1}$ в $L_{2}$ назьвается линейное инъективное отображение $J: L_{1} \rightarrow L_{2}$. 
ОпРЕДЕЛЕНИЕ. Вложение $J: H \rightarrow H_{1}$ назовем $\mathfrak{K}$ - вложсением, если существует изоморфизм $U: H_{1} \rightarrow H$ такой, что $U J \in \mathfrak{K}$.

Очевидно, что $\mathfrak{K}$-вложение является непрерьвньм.

Пусть $J: H \rightarrow H_{1}$ является $\mathfrak{K}$-вложением. Отождествим пространство $H$ с его образом при этом вложении. Для элементов из $H$ теперь определены две нормы, которые будем обозначать через $\|\cdot\|_{H}$ и $\|\cdot\|_{H_{1}}$ соответственно. Отметим, что для любой последовательности $\left\{x_{n}\right\} \in \ell_{0}^{\infty}(H, \mathfrak{K})$

$$
\lim _{n \rightarrow \infty}\left\|x_{n}\right\|_{H_{1}}=0
$$

Наложим последнее ограничение на идеал $\mathfrak{K}$ :

В) существует подмножество $\mathfrak{K}^{\prime} \subset \mathfrak{K}$, плотное в $\mathfrak{K}$ в равномерной операторной топологии, такое, что для любого оператора $A \in \mathfrak{K}^{\prime}$ найдется константа $c(=c(A))$ такая, что для всех $x \in H$

$$
\|A x\|_{H} \leqslant c \cdot\|x\|_{H_{1}} .
$$

В этих обозначениях и предположениях справедливо утверждение.

ТЕОрема 1. Предположим, что $J: H \rightarrow H_{1}$ является $\mathfrak{K}$-вложсением. Для оператора $A \in \mathfrak{A}$ следующие условия равносильнь:

1) смежсный класс $A+\mathfrak{K} \in \mathfrak{A} / \mathfrak{K}$ обратим слева;

2) выполняется априорная оценка

$$
\|x\|_{H} \leqslant \operatorname{const}\left(\|A x\|_{H}+\|x\|_{H_{1}}\right), \quad x \in H .
$$

ДокАЗАТЕльство. 1) $\Longrightarrow 2$ ). Выберемпроизвольный оператор $R$ из смежного класса, обратного слева к $A+\mathfrak{K}$. Тогда имеет место равенство $R A=I+T$, где $T \in \mathfrak{K}$. Воспользуемся свойством В). Выберем оператор $T_{0} \in \mathfrak{K}^{\prime}$, для которого $\left\|T-T_{0}\right\|<1 / 2$. Положим $\Delta=T-T_{0}$. Оператор $I+\Delta$ обратим и

$$
\left\|(I+\Delta)^{-1}\right\| \leqslant \frac{1}{1-\|\Delta\|} \leqslant 2 .
$$

Тогда, учитьвая равенство $I+\Delta=R A-T_{0}$, получаем

$$
\|x\|_{H} \leqslant\left\|(I+\Delta)^{-1}\right\| \cdot\left\|\left(R A-T_{0}\right) x\right\|_{H} \leqslant \operatorname{const}\left(\|A x\|_{H}+\|x\|_{H_{1}}\right)
$$

с константой, не зависящей от $x \in H$.

$2) \Longrightarrow 1$ ). Обратимость слева линейного непрерывного оператора $B$, действующего в гильбертовом пространстве $\mathscr{H}$, равносильна наличию оценки

$$
\|x\| \leqslant \text { const }\|B x\|, \quad x \in \mathscr{H} .
$$

Вьполнение оценки достаточно проверить на произвольном плотном в $\mathscr{H}$ множестве. Покажем, что из условия 2) теоремы вытекает оценка вида (4) для оператора $\widehat{A} \in \mathscr{B}(\mathbb{H})$. 
Пусть $\left\{x_{n}\right\} \in \ell_{0}^{\infty}(H, \mathfrak{K})$. Тогда

$$
\left\|x_{n}\right\|_{H} \leqslant \operatorname{const}\left(\left\|A x_{n}\right\|_{H}+\left\|x_{n}\right\|_{H_{1}}\right) .
$$

Применяя к обеим частям неравенства функционал Lim и учитьвая, что

$$
\operatorname{Lim}_{n \rightarrow \infty}\left\|x_{n}\right\|_{H_{1}}=\lim _{n \rightarrow \infty}\left\|x_{n}\right\|_{H_{1}}=0
$$

получаем

$$
\operatorname{Lim}_{n \rightarrow \infty}\left\|x_{n}\right\|_{H} \leqslant \text { const } \operatorname{Lim}_{n \rightarrow \infty}\left\|A x_{n}\right\|_{H} .
$$

Это означает, что для любого $\bar{x} \in \ell_{0}^{\infty}(H, \mathfrak{K}) / \mathfrak{N}$

$$
\|\bar{x}\| \leqslant \text { const }\|\widehat{A} \bar{x}\| \text {. }
$$

Тогда оператор $\widehat{A}$ обратим слева в $\mathscr{B}(\mathbb{H})$. Множество $\Xi(\mathfrak{A} / \mathfrak{K})$ является $C^{*}$-подалгеброй в $\mathscr{B}(\mathbb{H})$. В силу леммы 1 оператор $\widehat{A}$ обратим слева в $\Xi(\mathfrak{A} / \mathfrak{K})$. Тогда смежный класс $A+\mathfrak{K} \in \mathfrak{A} / \mathfrak{K}$ обратим слева. Теорема доказана.

СледСтвиЕ 1. Предположим, что $J: H \rightarrow H_{1}$ является $\mathfrak{K}$-вложением. Для оператора $A \in \mathfrak{A}$ следующие условия равносильны:

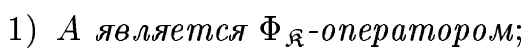

2) выполняются априорные оченки

$$
\begin{array}{ll}
\|x\|_{H} \leqslant \operatorname{const}\left(\|A x\|_{H}+\|x\|_{H_{1}}\right), & x \in H, \\
\|y\|_{H} \leqslant \operatorname{const}\left(\left\|A^{*} y\right\|_{H}+\|y\|_{H_{1}}\right), & y \in H .
\end{array}
$$

Мы будем использовать также модификацию теоремы 1. Рассмотрим следующее условие:

$\Gamma)$ в пространстве $H$ задана норма $\|\cdot\|_{1}$ такая, что для любой последовательности $\left\{x_{n}\right\} \in \ell_{0}^{\infty}(H, \mathfrak{K})$

$$
\lim _{n \rightarrow \infty}\|x\|_{1}=0
$$

Будем предполагать, что условие В) выполняется с заменой $\|\cdot\|_{H_{1}}$ на $\|\cdot\|_{1}$. Отметим, что именно условие вида $\Gamma$ ) (вытекающее из предположения о том, что $J$ является $\mathfrak{K}$-вложением) используется в доказательстве теоремы 1 . Тогда, добавляя к ограничениям на идеал $\mathfrak{K}$ условие $\Gamma$ ), можно снять условие о $\mathfrak{K}$-вложении. Мы получаем следующее утверждение.

СледСтвИЕ 2. Для оператора $A \in \mathfrak{A}$ следующие условия равносильнь:

1) смежный класс $A+\mathfrak{K} \in \mathfrak{A} / \mathfrak{K}$ обратим слева;

2) выполняется априорная оценка

$$
\|x\|_{H} \leqslant \operatorname{const}\left(\|A x\|_{H}+\|x\|_{1}\right), \quad x \in H .
$$


2. Перейдем теперь к приложениям теоремы 1.

Обозначим через $H \widehat{\otimes} H$ пополнение алгебраического тензорного произведения $H \otimes H$ по стандартной норме [5, с. 230]. Рассмотрим множество всех операторов из $\mathscr{B}(H \widehat{\otimes} H)$, представимых в виде конечных сумм $\sum_{i} A_{i} \otimes B_{i}$, где $A_{i} \in \mathscr{K}(H), B_{i} \in \mathscr{B}(H)$ для всех значений индекса $i$. Обозначим через $\mathscr{K}(H) \widehat{\otimes} \mathscr{B}(H)$ замыкание указанного множества в равномерной операторной топологии. Введем обозначение $\mathfrak{K}_{1}=\mathscr{K}(H) \widehat{\otimes} \mathscr{B}(H)$. Аналогично определим множества

$$
\mathfrak{A} \stackrel{\text { def }}{=} \mathscr{B}(H) \widehat{\otimes} \mathscr{B}(H), \quad \mathfrak{K}_{2} \stackrel{\text { def }}{=} \mathscr{B}(H) \widehat{\otimes} \mathscr{K}(H), \quad \mathfrak{K}_{0} \stackrel{\text { def }}{=} \mathscr{K}(H) \widehat{\otimes} \mathscr{K}(H) .
$$

Обозначим через $\mathfrak{K}_{12}$ алгебраическую сумму множеств $\mathfrak{K}_{1}$ и $\mathfrak{K}_{2}$. Тогда (см. [1, с. 284]) $\mathfrak{A}$ является $C^{*}$-подалгеброй алгебры $\mathscr{B}(H \widehat{\otimes} H)($ легко показать, что $\mathfrak{A} \neq \mathscr{B}(H \widehat{\otimes} H))$, $\mathfrak{K}_{0}, \mathfrak{K}_{1}, \mathfrak{K}_{2}, \mathfrak{K}_{12}$ суть собственные замкнутые двусторонние $*$-идеалы в $\mathfrak{A}, \mathfrak{K}_{1} \cap \mathfrak{K}_{2}=\mathfrak{K}_{0}=$ $\mathscr{K}(H \widehat{\otimes} H)$.

ОПрЕДЕлЕниЕ. Оператор $A \in \mathfrak{A}$ назовем $\Phi_{1}$-оператором, если смежньй класс $A+$ $\mathfrak{K}_{1} \in \mathfrak{A} / \mathfrak{K}_{1}$ обратим.

Аналогично с идеалами $\mathfrak{K}_{2}$ и $\mathfrak{K}_{12}$ связьваем понятия $\Phi_{2}$ - и $\Phi_{12}$-операторов.

Лемма 4. Oператор $A \in \mathfrak{A}$ является $n$-нормальным тогда и только тогда, когда смежнные классы

$$
A+\mathfrak{K}_{1} \in \mathfrak{A} / \mathfrak{K}_{1}, \quad A+\mathfrak{K}_{2} \in \mathfrak{A} / \mathfrak{K}_{2}
$$

обратимы слева.

ДокАЗАтЕльство. Предположим, что $A$ является $n$-нормальным. Из леммы 1 получаем, что смежный класс $A+\mathfrak{K}_{0} \in \mathfrak{A} / \mathfrak{K}_{0}$ обратим слева. Тогда из вложений $\mathfrak{K}_{0} \subset \mathfrak{K}_{1}$, $\mathfrak{K}_{0} \subset \mathfrak{K}_{2}$ вытекает обратимость смежных классов (5).

Допустим, что смежные классы (5) обратимы слева, $R_{1}$ и $R_{2}$ - представители соответствующих обратных смежных классов. Тогда

$$
R_{1} A=I+T_{1}, \quad R_{2} A=I+T_{2},
$$

где $T_{1} \in \mathfrak{K}_{1}, T_{2} \in \mathfrak{K}_{2}$. Рассмотрим оператор $R=R_{1}+R_{2}-R_{1} A R_{2}$. Учтем, что $R \in \mathfrak{A}$, $R A-I=-\left(R A_{1}-I\right)\left(R A_{2}-I\right)=-T_{1} T_{2}$. Поскольку $T_{1} T_{2} \in \mathfrak{K}_{1} \cap \mathfrak{K}_{2}\left(=\mathfrak{K}_{0}\right)$, отсюда выводим обратимость смежного класса $A+\mathfrak{K}_{0} \in \mathfrak{A} / \mathfrak{K}_{0}$ слева. Лемма доказана.

Из леммы 4 вытекает, что $d$-нормальность оператора $A \in \mathfrak{A}$ равносильна обратимости смежных классов (5) справа. Отсюда получаем следующее утверждение.

Лемма 5. Oператор $A \in \mathfrak{A}$ является Ф-оператором тогда и только тогда, когда он является $\Phi_{1}-u \Phi_{2}$-оператором.

Предположим, что $J: H \rightarrow H_{1}$ - компактное вложение. Покажем, что пространства $H \widehat{\otimes} H, H_{1} \widehat{\otimes} H$, вложение

$$
J_{1} \stackrel{\text { def }}{=} J \otimes I: H \widehat{\otimes} H \rightarrow H_{1} \widehat{\otimes} H,
$$

алгебра $\mathfrak{A}$ и ее идеал $\mathfrak{K}_{1}$ удовлетворяют всем требованиям теоремы 1. 
Пусть $U: H_{1} \rightarrow H$ - изоморфизм пространств $H_{1}$ и $H$. Тогда $U \otimes I: H_{1} \widehat{\otimes} H \rightarrow H \widehat{\otimes} H$ является изоморфизмом пространств $H_{1} \widehat{\otimes} H$ и $H \widehat{\otimes} H$. Кроме того, $(U \otimes I) J_{1}=U J \otimes I$ $\in \mathfrak{K}_{1}$, так как $U J \in \mathfrak{K}(H)$, т.е. $J_{1}$ является $\mathfrak{K}_{1}$-вложением. Перейдем теперь к проверке условий А)-В).

Пусть $\left\{P_{n}\right\}, n=1,2, \ldots,-$ последовательность конечномерных ортопроекторов в $H \widehat{\otimes} H$, сильно сходящаяся к единичному оператору. Для любого оператора $T \in \mathfrak{K}(H)$

$$
\lim _{n \rightarrow \infty}\left\|T-T P_{n}\right\|=0
$$

Отсюда вытекает такое утверждение.

Лемма 6. Для оператора $A \in \mathfrak{A}$ следуюшие условия равносильны:

1) $A \in \mathfrak{K}_{1}$;

2) $\lim _{n \rightarrow \infty}\left\|A-A\left(P_{n} \otimes I\right)\right\|=0$.

Полагая $R_{n}=P_{n} \widehat{\otimes} I, n=1,2, \ldots$, получаем, что для идеала $\mathfrak{K}_{1}$ вьполнено условие Б). При $n \rightarrow \infty$ операторы $R_{n}$ сильно сходятся к единичному оператору. Поэтому для идеала $\mathfrak{K}_{1}$ вьполнено условие $\left.\mathrm{A}\right)$.

Перейдем к проверке условия В). Оператор $J^{*}: H_{1} \rightarrow H$ имеет плотный в пространстве $H$ образ, поскольку ker $J=\{0\}$. Рассмотрим множество всех действующих в пространстве $H$ операторов вида

$$
T x=u(x, v)_{H}, \quad x \in H,
$$

где $u \in H, v \in \operatorname{im} J^{*}$.

Пусть $v=J^{*} w, w \in H_{1}$. Тогда

$$
\begin{aligned}
\|T x\|_{H} & =\|u\|_{H}\left|\left(x, J^{*} w\right)_{H}\right|=\|u\|_{H}\left|(J x, w)_{H_{1}}\right| \\
& \leqslant\|u\|_{H}\|w\|_{H_{1}}\|J x\|_{H_{1}}=\|u\|_{H}\|w\|_{H_{1}}\|x\|_{H_{1}} \leqslant \mathrm{const}\|x\|_{H_{1}}, \quad x \in H .
\end{aligned}
$$

Для оператора вида (6) имеет место оценка $\|T\|_{H \rightarrow H} \leqslant\|u\|_{H}\|v\|_{H}$. Из нее следует, что любой одномерньй оператор в $H$ (имеющий вид (6) с произвольным элементом $v \in H$ ) может быть приближен по норме операторами вида (6). Поэтому любой конечномерньй оператор, действующий в пространстве $H$, может быть приближен конечными суммами операторов вида (6). Тогда этим свойством обладает и любой компактньй оператор в $H$.

Обозначим через $\mathfrak{K}_{1}^{\prime}$ множество всех действующих в пространстве $H \widehat{\otimes} H$ операторов, представимых в виде конечных сумм

$$
\sum_{i} T_{i} \otimes B_{i}
$$

где для всех значений индекса $i$ операторы $T_{i}$ имеют вид $(6), B_{i} \in \mathscr{B}(H)$.

В силу сказанного множество $\mathfrak{K}_{1}^{\prime}$ плотно в $\mathfrak{K}_{1}$ в равномерной операторной топологии. Из (7) получаем, что для любого $A \in \mathfrak{K}_{1}^{\prime}$ вьполняется оценка

$$
\|A x\|_{H \widehat{\otimes} H} \leqslant \text { const }\|x\|_{H_{1} \widehat{\otimes} H}, \quad x \in H \widehat{\otimes} H .
$$


Мы доказали, что вьполняется условие В).

В приводимой ниже теореме 2 используются следующие априорные оценки:

$$
\begin{array}{ll}
\|x\|_{H \widehat{\otimes} H} \leqslant \operatorname{const}\left(\|A x\|_{H \widehat{\otimes} H}+\|x\|_{H_{1} \widehat{\otimes} H}\right), & x \in H \widehat{\otimes} H, \\
\|y\|_{H \widehat{\otimes} H} \leqslant \operatorname{const}\left(\left\|A^{*} y\right\|_{H \widehat{\otimes} H}+\|y\|_{H_{1} \widehat{\otimes} H}\right), & y \in H \widehat{\otimes} H, \\
\|x\|_{H \widehat{\otimes} H} \leqslant \operatorname{const}\left(\|A x\|_{H \widehat{\otimes} H}+\|x\|_{H \widehat{\otimes} H_{1}}\right), & x \in H \widehat{\otimes} H, \\
\|y\|_{H \widehat{\otimes} H} \leqslant \operatorname{const}\left(\left\|A^{*} y\right\|_{H \widehat{\otimes} H}+\|y\|_{H \widehat{\otimes} H_{1}}\right), & y \in H \widehat{\otimes} H .
\end{array}
$$

Из теоремы 1 , лемм 4, 5 и приведенных выше построений получаем следующее утверждение.

Tеорема 2. Пусть $A \in \mathfrak{A}$. Смежсный класс $A+\mathfrak{K}_{1} \in \mathfrak{A} / \mathfrak{K}_{1}$ обратим слева (справа) тогда и только тогда, когда выполняется оценка (8) (оценка (9)). Oператор $A$ является $\Phi_{1}$-оператором ( $\Phi_{2}$-оператором) тогда и только тогда, когда выполняются оценки (8), (9) (оченки (10), (11)). Оператор А является п-нормальным ( $\kappa и(8),(10)$ (оценки (9), (11)). Оператор А является Ф-оператором тогда и только тогда, когда выполняются оченки (8)-(11).

Перейдем теперь к идеалу $\mathfrak{K}_{12}$. Здесь, как и вьше, предполагается, что пространство $H$ компактно вложено в пространство $H_{1}$. Мы воспользуемся следствием 2 из теоремы 1.

Определим в пространстве $H \widehat{\otimes} H$ норму

$$
\|x\|_{12}=\|x\|_{H_{1} \widehat{\otimes} H}+\|x\|_{H \widehat{\otimes} H_{1}} .
$$

Покажем, что алгебра $\mathfrak{A}=\mathscr{B}(H) \widehat{\otimes} \mathscr{B}(H)$, идеал $\mathfrak{K}_{12}$ и эта норма удовлетворяют условиям А)-Г).

Рассмотрим последовательность операторов

$$
R_{n}=(I \otimes I)-\left(I-P_{n}\right) \otimes\left(I-P_{n}\right), \quad n=1,2, \ldots,
$$

действуюших в пространстве $H \widehat{\otimes} H$, где $\left\{P_{n}\right\}$ - введенная вьше последовательность конечномерных ортопроекторов. Легко видеть, что для каждого $n$ оператор $R_{n}$ является ортопроектором и при $n \rightarrow \infty$ операторы $R_{n}$ сильно сходятся к единичному оператору из $\mathscr{B}(H \widehat{\otimes} H)$. Покажем, что вьполняется условие Б).

Пусть $A=T \otimes B$, где $T \in \mathscr{K}(H), B \in \mathscr{B}(H)$. Тогда

$$
\left\|A-A R_{n}\right\|=\left\|T\left(I-P_{n}\right)\right\| \cdot\left\|B\left(I-P_{n}\right)\right\| \leqslant\left\|T\left(I-P_{n}\right)\right\| \cdot\|B\| \rightarrow 0
$$

при $n \rightarrow \infty$. Из симметричных соображений получаем, что требуемое равенство выполняется для оператора $B \otimes T$ с сомножителями указанного вида. Поскольку эти операторы образуют в $\mathfrak{K}_{12}$ полное множество, доказьваемое равенство вьполняется для всех операторов из $\mathfrak{K}_{12}$.

Перейдем к свойству В) (с заменой $H$ на $H \widehat{\otimes} H, \mathfrak{K}$ на $\mathfrak{K}_{12}$ и $\|\cdot\|_{H_{1}}$ на $\left.\|\cdot\|_{12}\right)$. Рассмотрим множество $\mathfrak{K}_{12}^{\prime}$ всех операторов в $H \widehat{\otimes} H$, представимых в виде конечных сумм

$$
\sum_{i} T_{i} \otimes B_{i}+\sum_{j} B_{j}^{\prime} \otimes T_{j}^{\prime},
$$


где для всех значений индексов $i, j$ операторы $T_{i}, T_{j}^{\prime}$ имеют вид $(6), B_{i}, B_{j}^{\prime} \in \mathscr{B}(H)$.

Учитывая результаты, полученные выше при доказательстве свойства В) для идеала $\mathfrak{K}_{1}$, и симметричные результаты для $\mathfrak{K}_{2}$, получаем, что это свойство выполняется и для $\mathfrak{K}_{12}$.

Перейдем к свойству $\Gamma)$. Пусть $\left\{x_{n}\right\} \in \ell^{\infty}\left(H \widehat{\otimes} H, \mathfrak{K}_{12}\right)$. Тогда $\left\{x_{n}\right\} \in \ell^{\infty}\left(H \widehat{\otimes} H, \mathfrak{K}_{1}\right)$. Снова учитьвая полученный результат для $\mathfrak{K}_{1}$, вьводим, что

$$
\lim _{n \rightarrow \infty}\left\|x_{n}\right\|_{H_{1} \widehat{\otimes} H}=0
$$

Из симметричных соображений следует, что

$$
\lim _{n \rightarrow \infty}\left\|x_{n}\right\|_{H \widehat{\otimes} H_{1}}=0
$$

т.e.

$$
\lim _{n \rightarrow \infty}\left\|x_{n}\right\|_{12}=0
$$

В формулировке теоремы 3 используются следующие априорные оценки для оператора $A \in \mathscr{B}(H \widehat{\otimes} H)$ :

$$
\begin{array}{ll}
\|x\|_{H \widehat{\otimes} H} \leqslant \operatorname{const}\left(\|A x\|_{H \widehat{\otimes} H}+\|x\|_{H_{1} \widehat{\otimes} H}+\|x\|_{H \widehat{\otimes} H_{1}}\right), & x \in H \widehat{\otimes} H, \\
\|y\|_{H \widehat{\otimes} H} \leqslant \operatorname{const}\left(\left\|A^{*} y\right\|_{H \widehat{\otimes} H}+\|y\|_{H_{1} \widehat{\otimes} H}+\|y\|_{H \widehat{\otimes} H_{1}}\right), & y \in H \widehat{\otimes} H .
\end{array}
$$

Согласно следствию 2 из теоремы 1 получаем такое утверждение.

Теорема 3. Пусть $A \in \mathfrak{A}$. Смежинй класс $A+\mathfrak{K}_{12} \in \mathfrak{A} / \mathfrak{K}_{12}$ обратим слева (справа) тогда и только тогда, когда выполняется оченка (12) (оченка (13)). Oператор $A$ является $\Phi_{12}$-оператором тогда и только тогда, когда выполняются оченки (12) и (13).

Автор признателен В. М. Деундяку за полезное обсуждение работы.

\section{СПИСОК ЦИТИРОВАННОЙ ЛИТЕРАТУРЫ}

[1] Крейн С. Г. Линейные уравнения в банаховом пространстве. М.: Наука, 1971.

[2] Cordes H. O. On a class of $C^{*}$-algebras // Math. Ann. 1967. V. 170. № 4. P. 283-313.

[3] Пилиди В. С. О многомерных бисингулярных операторах // Докл. АН СССР. 1971. Т. 201. №1. C. $787-789$.

[4] Calkin J. W. Two-sided ideals and congruences in the ring of bounded operators // Ann. of Math. 1941. V. 42. № 2. P. 839-873.

[5] Мёрфи Дж. $C^{*}$-алгебры и теория операторов. М.: Факториал, 1997.

[6] Эдвардс Р. Функциональный анализ. Теория и приложения. М.: Мир, 1968. 